\title{
Semilocal density functional theory with correct surface asymptotics
}

\author{
Lucian A. Constantin, ${ }^{1}$ Eduardo Fabiano, ${ }^{2,1}$ J. M. Pitarke, ${ }^{3,4}$ and Fabio Della Sala ${ }^{2,1}$ \\ ${ }^{1}$ Center for Biomolecular Nanotechnologies @ UNILE, Istituto Italiano di Tecnologia, Via Barsanti, I-73010 Arnesano, Italy \\ ${ }^{2}$ Istituto Nanoscienze-CNR, Euromediterranean Center for Nanomaterial Modelling and Technology (ECMT), Via per Arnesano, \\ 73100 Lecce, Italy \\ ${ }^{3}$ CIC nanoGUNE, Tolosa Hiribidea 76, E-20018 Donostia, Basque Country \\ ${ }^{4}$ Materia Kondentsatuaren Fisika Saila, DIPC, and Centro Física Materiales CSIC-UPV/EHU, 644 Posta kutxatila, \\ E-48080 Bilbo, Basque Country
}

(Received 16 December 2015; revised manuscript received 27 February 2016; published 16 March 2016)

\begin{abstract}
Semilocal density functional theory is the most used computational method for electronic structure calculations in theoretical solid-state physics and quantum chemistry of large systems, providing good accuracy with a very attractive computational cost. Nevertheless, because of the nonlocality of the exchange-correlation hole outside a metal surface, it was always considered inappropriate to describe the correct surface asymptotics. Here, we derive, within the semilocal density functional theory formalism, an exact condition for the imagelike surface asymptotics of both the exchange-correlation energy per particle and potential. We show that this condition can be easily incorporated into a practical computational tool, at the simple meta-generalized-gradient approximation level of theory. Using this tool, we also show that the Airy-gas model exhibits asymptotic properties that are closely related to those at metal surfaces. This result highlights the relevance of the linear effective potential model to the metal surface asymptotics.
\end{abstract}

DOI: 10.1103/PhysRevB.93.115127

\section{INTRODUCTION}

The exact form of the potential felt by an electron leaving from or approaching a metal surface is of great importance for a variety of physical phenomena, including the interpretation of image states [1], modeling quantum transport [2], lowenergy electron diffraction (LEED) [3], scanning tunneling microscopy [4,5], and inverse or two-photon photoemission spectroscopy $[6,7]$. The asymptotic form of this image potential is $-1 /\left[4\left(z-z_{0}\right)\right]$, with $z$ being the distance from the surface, and $z_{0}$ representing the position of the so-called image plane [8], and should be reproduced by any computational method aiming at an accurate description of the surface physics.

Within Kohn-Sham (KS) density functional theory (DFT) $[9,10]$, which is the most used computational method for electronic structure calculations in theoretical solid-state physics, the shape of the image potential is dictated by the properties of the effective Kohn-Sham (KS) potential. This depends on the employed approximation for the exchange-correlation (xc) functional $E_{\mathrm{xc}}[\rho]$, which gives the xc potential via the relation

$$
v_{\mathrm{xc}}(\mathbf{r})=\frac{\delta E_{\mathrm{xc}}[\rho]}{\delta \rho(\mathbf{r})},
$$

where $\rho$ is the electron density. It has been shown that the exact $v_{\text {xc }}$ asymptotically approaches the image potential [8,11-14], despite a different result has been obtained within the plasmonpole approximation [15].

The popular local density approximation (LDA) [10] and the generalized gradient approximation (GGA), however, fail in this task [16], showing either a too fast decay (e.g., exponential), or an inaccurate description of the surface energetics (as for the Becke exchange [17]). Ad hoc modifications of the LDA xc potential $[18,19]$ have then been proposed to improve the asymptotic behavior of the $\mathrm{xc}$ potential, but such methods are not functional derivatives of any energy functional. Alternatively, nonlocal methods outside the KS framework [11,13,14,20-22] are employed.

An accurate KS-DFT method with the correct surface asymptotics would be desirable for many reasons, including the local nature and the computational efficiency. However, a good functional shall yield not only the correct asymptotic xc potential, but also accurate energies. Thus, it is necessary to be defined by a realistic energy per particle $\varepsilon_{\mathrm{xc}}(\mathbf{r})$. The latter is not a uniquely defined physical quantity, but an exact reference for it is the conventional energy per particle, which is associated with the interaction of an electron at $\mathbf{r}$ with the coupling-constant-averaged charge of its xc hole [23-25]. The exact (conventional) $\varepsilon_{\mathrm{xc}}(\mathbf{r})$ at metal surfaces decays as $-1 /\left[4\left(z-z_{0}\right)\right]$, i.e., as the image potential $[11,26]$.

We recall that the exact exchange energy per particle decreases as $\epsilon_{x}(z \rightarrow \infty) \rightarrow-A(\beta) / z$ where $A(\beta)=[\pi+$ $2 \beta \ln (\beta)] /\left[2 \pi\left(1+\beta^{2}\right)\right], \beta=\sqrt{\epsilon_{F} / W}, \epsilon_{F}=k_{F}^{2} / 2$ being the Fermi energy (and $k_{F}$ the Fermi wave vector), and $W$ the work function $[27,28]$. On the other hand, the exact exchange potential behaves as $v_{x}(z \rightarrow \infty) \rightarrow \ln \left(\beta k_{F} z\right) /(2 \pi \beta z)$ [29]. Note that these behaviors are related to semi-infinite surfaces; for finite jellium slabs we have that, as in molecules, $\epsilon_{x}(z \rightarrow$ $\infty)=-1 /(2 z)$ [27] and $v_{x}(z \rightarrow \infty)=-1 /(z)$ [30-34].

The simultaneous description of surface asymptotic and energy properties is anyway an ambitious objective, which is in fact not achievable at the GGA level [35-38]. In this paper, we show that the issue can be instead solved at the meta-GGA level of theory, employing an exact condition which yields the correct imagelike asymptotic behavior of both $\varepsilon_{\mathrm{xc}}$ and $v_{\mathrm{xc}}$ at metal surfaces. This condition can be easily implemented in any meta-GGA functional, keeping its original accuracy for ground-state properties not related to surface asymptotics. Hence, an accurate KS-DFT method with correct metal-surface asymptotic can be obtained for application in many surface science problems. 


\section{EXACT CONDITION FOR ASYMPTOTIC PROPERTIES}

To start, we consider the simplest (and most used) model for a metal surface: the semi-infinite jellium surface. This model system is very important in surface science and solidstate physics, containing the physics of simple metal surfaces $[8,39,40]$.

The KS single-particle orbitals have the form

$$
\Psi_{k_{z}, \mathbf{k}_{\|}}(\mathbf{r})=\frac{1}{\sqrt{S} \sqrt{L}} e^{i \mathbf{k}_{\|} \mathbf{r}_{\|}} \phi_{k_{z}}(z),
$$

where $\mathbf{k}_{\|}$and $\mathbf{r}_{\|}$are the two-dimensional wave vector and position vector in the plane $x y$ of the surface, $k_{z}$ and $z$ are the corresponding components in the direction perpendicular to the surface, $S$ and $L$ are the normalization area and length, and $\phi_{k_{z}}(z)$ are the eigenfunctions of a one-dimensional KS Hamiltonian (for details see Appendix A).

In the vacuum region, far away from the surface $(z \rightarrow \infty)$, the single-particle orbitals $\phi_{k_{z}}(z)$ behave as [27]

$$
\phi_{k_{z}}(z \rightarrow \infty) \rightarrow \phi_{k_{F}}(z \rightarrow \infty) e^{-\beta z\left(k_{F}-k_{z}\right)},
$$

where

$$
\phi_{k_{F}}(z \rightarrow \infty) \sim e^{-z \sqrt{2 W}}(2 z \sqrt{2 W})^{\alpha_{K S} / \sqrt{2 W}},
$$

$\alpha_{\mathrm{KS}}>0$.

Now, we consider a meta-GGA xc energy per particle of the form

$$
\epsilon_{\mathrm{xc}}^{\mathrm{MGGA}}=\epsilon_{x}^{\mathrm{LDA}} \frac{1}{\eta} \frac{8 \pi}{3 \sqrt{5}} \frac{\sqrt{\alpha}}{\sqrt{\ln (\alpha)}},
$$

where $\eta$ is a parameter to be fixed later, $\alpha(\mathbf{r})=$ $\left[\tau(\mathbf{r})-\tau^{W}(\mathbf{r})\right] / \tau^{\mathrm{TF}}(\mathbf{r})$ is the well-known meta-GGA ingredient that measures the nonlocality of the kinetic-energy density [38], with $\tau, \tau^{W}$, and $\tau^{\mathrm{TF}}$ being the positive-defined exact $\mathrm{KS}$, von Weizsäcker, and Thomas-Fermi kinetic-energy densities, respectively. We recall that $1 /\left[1+\alpha(\mathbf{r})^{2}\right]$ is the electron localization function, often used in the characterization of chemical bonds [41]. Equation (5) yields the following asymptotic behaviors (see the Appendix B for details):

$$
\begin{aligned}
& \epsilon_{\mathrm{xc}}^{\mathrm{MGGA}}(z \rightarrow \infty) \rightarrow-\frac{1}{\eta} \frac{1}{z}+O\left(z^{-2}\right), \\
& v_{\mathrm{xc}}^{\mathrm{MGGA}}(z \rightarrow \infty) \rightarrow-\frac{3}{2 \eta} \frac{1}{z}+O\left(z^{-2}\right) .
\end{aligned}
$$

Here, the KS potential has been obtained in the generalized KS framework using the formula [42]

$$
\begin{aligned}
v_{\mathrm{xc}}(\mathbf{r}) \Psi_{i}(\mathbf{r})= & {\left[\frac{\partial\left(\rho \epsilon_{\mathrm{xc}}\right)}{\partial \rho}-\nabla \frac{\partial\left(\rho \epsilon_{\mathrm{xc}}\right)}{\partial \nabla \rho}\right] \Psi_{i} } \\
& -\frac{1}{2} \nabla\left(\frac{\partial\left(\rho \epsilon_{\mathrm{xc}}\right)}{\partial \tau}\right) \nabla \Psi_{i}-\frac{1}{2} \frac{\partial\left(\rho \epsilon_{\mathrm{xc}}\right)}{\partial \tau} \nabla^{2} \Psi_{i} .
\end{aligned}
$$

Equations (6) and (7) show that, in contrast to previously developed xc functionals, both $v_{\mathrm{xc}}$ and $\epsilon_{\mathrm{xc}}$ are proportional to the exact ones: if $\eta=\eta_{1}=4\left(\eta=\eta_{2}=6\right)$ then the exact energy density (potential) is obtained. Unfortunately, $\eta_{1} \neq \eta_{2}$. Nevertheless, for both values Eq. (5) yields an asymptotic behavior qualitatively and quantitatively significantly beyond the current state of the art. We also remark that Eq. (5) is solely based on the properties of the reduced kinetic ingredient $\alpha$. However, at the meta-GGA level of theory, other ingredients are also available (e.g., the gradient and the Laplacian of the density) so that the exact asymptotic description of both $\epsilon_{\mathrm{xc}}$ and $v_{\mathrm{xc}}$ might be achieved.

\section{PRACTICAL COMPUTATIONAL TOOL}

As a first practical example, we consider the case $\eta=\eta_{1}$ and incorporate the condition of Eq. (5) into the popular TaoPerdew-Staroverov-Scuseria (TPSS) meta-GGA functional [43], using an approach similar to that of Ref. [44]. The resulting $\mathrm{xc}$ functional will be termed surface-asymptotics (SA) TPSS. This functional is obtained by simply changing, in the TPSS exchange formula, the parameter $\kappa$ (which determines the asymptotic behavior of the functional) from its original value of 0.804 to

$$
\kappa=\frac{2 \pi}{3 \sqrt{5}} \frac{\sqrt{\alpha+1}}{\sqrt{a+\ln (\alpha+b)}} .
$$

The correlation is left unchanged. (Note that the TPSS correlation decays exponentially, and thus our xc condition is incorporated in the TPSS exchange functional. This is a common procedure for semilocal functionals, which are based on a strong error cancellation between the exchange and correlation parts.) In Eq. (9), the parameters $a=2.413$ and $b=0.348$ have been fixed by imposing the constraints $\kappa=0.804$ for $\alpha=1$ and 0 ; whereas, when degenerate orbitals contribute to the tail of the density $(\alpha \rightarrow \infty), \kappa \rightarrow \frac{2 \pi}{3 \sqrt{5}} \frac{\sqrt{\alpha}}{\sqrt{\ln (\alpha)}}$ [i.e., Eq. (5)]. These conditions assure that (i) all the exact constraints satisfied by the original TPSS exchange functional are preserved and (ii) the new functional yields the correct imagelike asymptotics. The SA-TPSS functional does not recover locally the Lieb-Oxford bound [44], as TPSS does, but it satisfies the global Lieb-Oxford bound for all known physical systems (e.g., for atoms, molecules, solids, and surfaces $\left.E_{\mathrm{xc}}^{\mathrm{SA}-T P S S} \approx E_{\mathrm{xc}}^{\mathrm{TPSS}}\right)$. Moreover, it fulfills locally the simplified version of the Lewin-Lieb bound [see Eq. (22) of Ref. [45]].

In Fig. 1, we show the TPSS and SA-TPSS exchange enhancement factors versus $\alpha$ for several values of the reduced gradient $s=|\nabla \rho| /\left[2\left(3 \pi^{2}\right)^{1 / 3} \rho^{4 / 3}\right]$. When $s$ is small, TPSS and SA-TPSS coincide for all values of $\alpha$. As $s$ increases,

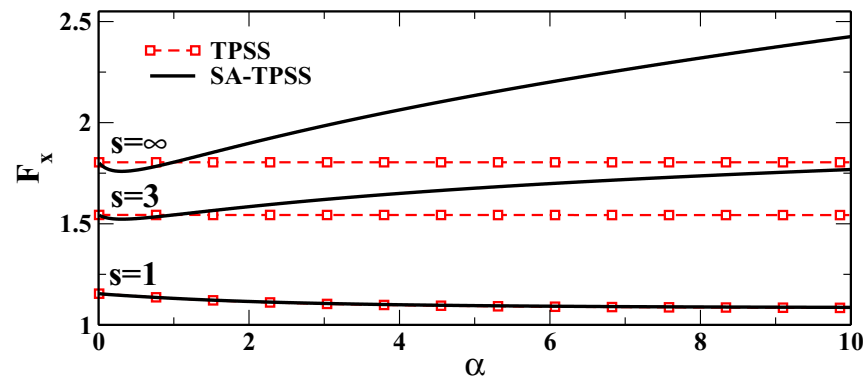

FIG. 1. TPSS and SA-TPSS exchange enhancement factors versus $\alpha$, for three values of the reduced gradient $s$. 


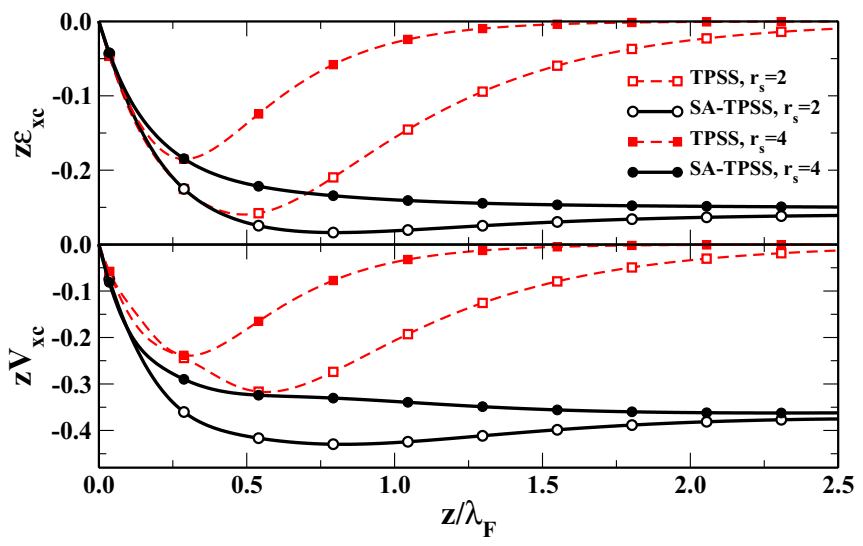

FIG. 2. $z \epsilon_{\mathrm{xc}}(z)$ (upper panel) and $z v_{\mathrm{xc}}(z)$ (lower panel) versus the reduced distance $z / \lambda_{F}$ ( $\lambda_{F}$ is the Fermi wavelength), for a jellium surface with electron-density parameter $r_{s}=2$ and 4 . The surface is located at $z=0$. Note that SA-TPSS gives $z \epsilon_{x c}=-\frac{1}{4}$ and $z v_{\mathrm{xc}}=-\frac{3}{8}$ far outside the surface.

TPSS and SA-TPSS start to differ, especially at large values of $\alpha$, as expected.

In Fig. 2, fully self-consistent KS-LDA orbitals were used to obtain $z \epsilon_{\mathrm{xc}}(z)$ (upper panel) and $z v_{\mathrm{xc}}(z)$ (lower panel) as a function of the scaled distance $z$ for jellium surfaces with the electron-density parameters $r_{s}=2$ and 4 . The TPSS functional yields a wrong (exponential) asymptotic behavior for both quantities. Instead, the SA-TPSS functional gives the following asymptotic behaviors: $\epsilon_{\mathrm{xc}}(z) \rightarrow-0.25 / z$ and $v_{\mathrm{xc}}(z) \rightarrow-0.375 / z$. Thus, Fig. 2 provides a numerical proof of the validity of Eq. (5), as well as a validation of the simple construction used to obtain the SA-TPSS functional.

In Tables I and II, we report the TPSS and SA-TPSS results for surface energies and various molecular tests, respectively. By construction, whenever the surface asymptotics plays a negligible role (e.g., covalent interactions in molecules: W4, OMRE, IP13, MGBL19) both functionals yield very similar results. In the case of jellium surface energies and noncovalent interactions (DI6, HB6), however, SA-TPSS improves over the standard TPSS.

Finally, we have to stress that the SA-TPSS meta-GGA gives only the correct asymptotic decay of the xc energy per particle and potential at metal surfaces, but it can not provide the exact behavior for the exchange and correlation

TABLE I. Jellium surface energies (in $\mathrm{erg} / \mathrm{cm}^{2}$ ), as obtained by using the functionals TPPS and SA-TPSS for various values of the electron-density parameter $r_{s}$. Diffusion Monte Carlo (DMC) calculations [46] are given as a reference. The results of the popular LDA [10,47] and PBE [48] functionals are also shown for comparison.

\begin{tabular}{lllllc}
\hline \hline$r_{s}$ & TPSS & SA-TPSS & LDA & PBE & Ref. \\
\hline 2 & 3380 & 3368 & 3354 & 3265 & $3392 \pm 50$ \\
3 & 772 & 767 & 764 & 741 & $768 \pm 10$ \\
4 & 266 & 263 & 261 & 252 & $261 \pm 8$ \\
6 & 55.5 & 54.5 & 53 & 52 & $53 \pm \ldots$ \\
\hline \hline
\end{tabular}

TABLE II. Mean absolute errors (in kcal/mol for energetical tests and in $\mathrm{m} \AA$ for the bond length test) of several molecular properties $[49,50]$.

\begin{tabular}{lccrr}
\hline \hline Test & TPSS & SA-TPSS & LDA & PBE \\
\hline Atomization energies (W4 test) & 4.7 & 4.8 & 44.0 & 10.7 \\
Reaction energies (OMRE test) & 8.0 & 7.9 & 21.0 & 6.7 \\
Ionization potentials (IP13 test) & 3.1 & 3.1 & 4.9 & 3.0 \\
Bond lengths (MGBL19 test) & 6.9 & 7.0 & 10.0 & 9.3 \\
Dipole interactions (DI6 test) & 0.6 & 0.4 & 2.7 & 0.4 \\
Hydrogen bonds (HB6 test) & 0.6 & 0.4 & 4.5 & 0.4 \\
\hline \hline
\end{tabular}

components, separately. This is a difficult task that, in our opinion, a simple semilocal functional can not obey.

\section{AIRY-GAS ASYMPTOTIC PROPERTIES}

As an additional example of the use of Eq. (5) and of the SA-TPSS functional, we consider the Airy gas [51,52], which is the simplest possible model for an edge electron gas (for details see Appendix A). This model system plays an important role in DFT [52-54], as it incorporates the correct physics of a semi-infinite metal surface and is simple enough to allow for analytical calculations. To our knowledge, the exact asymptotic behavior of the xc energy per particle and of KS xc potential of the Airy gas are unknown. Nevertheless, we can use the SA-TPSS functional to obtain some information about them.

The Airy-gas electron density and positive-defined kineticenergy density are $[51-53,55]$

$$
\begin{gathered}
\rho(z)=\frac{1}{3 \pi}\left[z^{2} \operatorname{Ai}^{2}(z)-z \operatorname{Ai}^{\prime 2}(z)-\operatorname{Ai}(z) \operatorname{Ai}^{\prime}(z) / 2\right], \\
\tau=-\frac{3}{10} z \rho(z)+\frac{1}{5} \rho^{\prime \prime}(z),
\end{gathered}
$$

where $z$ is the scaled distance and $\operatorname{Ai}(z)$ is the Airy function. In Fig. 3, we show the Airy-gas semilocal ingredient $s$, the reduced Laplacian $q=\nabla^{2} \rho /\left[4\left(3 \pi^{2}\right)^{2 / 3} \rho^{5 / 3}\right]$, and $\alpha$. In the bulk $(z \rightarrow-\infty)$, both $s$ and $q$ are small, while $\alpha \rightarrow 1$ (showing that the Thomas-Fermi theory becomes exact). In the vacuum, all semilocal ingredients diverge (as in the case of the jellium surface).

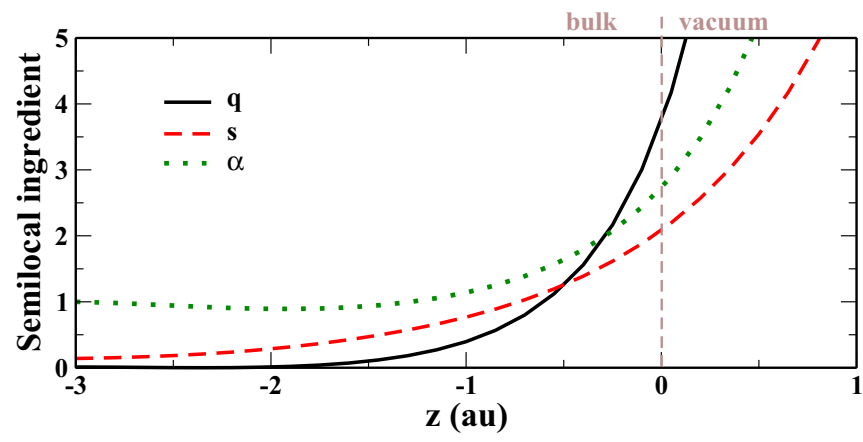

FIG. 3. The Airy-gas semilocal ingredients $(s, q$, and $\alpha)$ as a function of the scaled distance $z$. The Airy bulk is at $z \leqslant 0$. The exponential decay of the electron density occurs at $z \geqslant 0$. 


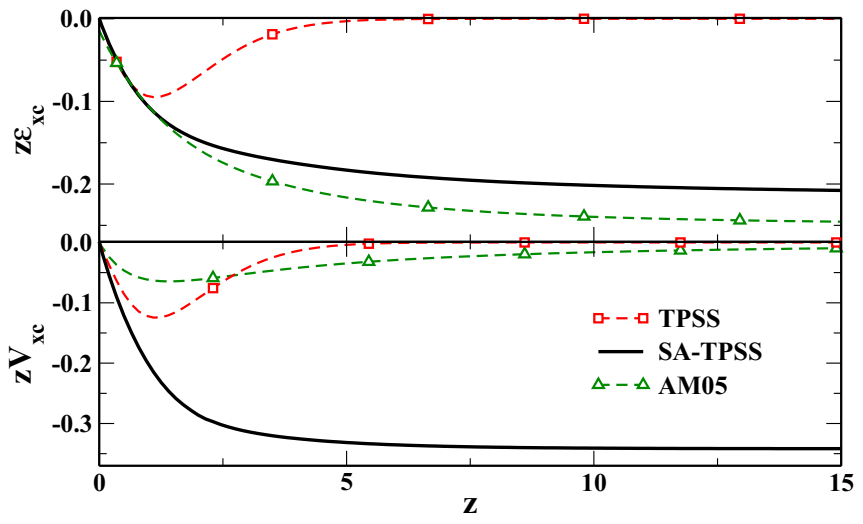

FIG. 4. The Airy-gas $z \epsilon_{\mathrm{xc}}(z)$ (upper panel) and $z v_{\mathrm{xc}}(z)$ (lower panel), as a function of the scaled distance $z$.

In the limit $z \rightarrow \infty$, the Airy-gas electron density and kinetic-energy density are

$$
\begin{aligned}
& \rho(z \rightarrow \infty) \rightarrow \frac{1}{32} \frac{e^{-4 / 3 z^{3 / 2}}}{\pi^{2} z^{3 / 2}}-\frac{35}{768} \frac{e^{-4 / 3 z^{3 / 2}}}{\pi^{2} z^{3}}+\ldots, \\
& \tau(z \rightarrow \infty) \rightarrow \frac{1}{64} \frac{e^{-4 / 3 z^{3 / 2}}}{\sqrt{z} \pi^{2}}+\frac{13}{1536} \frac{e^{-4 / 3 z^{3 / 2}}}{\pi^{2} z^{2}}+\ldots .
\end{aligned}
$$

The SA-TPSS xc functional gives the following analytical expressions:

$$
\begin{aligned}
& \epsilon_{\mathrm{xc}}^{\mathrm{SA}-T P S S}(z \rightarrow \infty) \rightarrow-\sqrt{3} /(8 z) \approx-0.217 / z, \\
& v_{\mathrm{xc}}^{\mathrm{SA-TPSS}}(z \rightarrow \infty) \rightarrow-3 \sqrt{3} /(16 z) \approx-0.325 / z .
\end{aligned}
$$

This result is interesting since it suggests that, for the Airy gas, both $\epsilon_{\mathrm{xc}}$ and $v_{\mathrm{xc}}$ decay as $-1 / z$, as in the case of the jellium surface. Furthermore, because the coefficients $(0.217$ and 0.325$)$ are close to, but smaller than, the ones for the jellium surface $(0.25$ and 0.375$)$, we can conclude that at a metal surface the main contribution to the asymptotics comes from the region near the surface, where the effective potential is linear and well described by the Airy-gas model. We note that such a result cannot be obtained without the proper inclusion of exact surface conditions into the functional. This is shown in Fig. 4 where we plot, for several functionals, $z \epsilon_{\mathrm{xc}}(z)$ (upper panel) and $z v_{\mathrm{xc}}(z)$ (lower panel), versus the scaled distance $z$, in the vacuum region of the Airy gas.

The TPSS functional shows a rather unphysical exponential decay for both $\varepsilon_{\mathrm{xc}}$ and $v_{\mathrm{xc}}$. On the other hand, the AM05 functional [52], whose energy density is fitted to the Airy gas, is close to our SA-TPSS functional for $\epsilon_{\mathrm{xc}}$ but displays a decay that is too fast for $v_{\mathrm{xc}}$. This latter feature represents a manifestation of the impossibility, at the GGA level, to describe correctly both $\epsilon_{\mathrm{xc}}$ and $v_{\mathrm{xc}}$, which can only be overcome at the meta-GGA level [38].

The exchange-only asymptotic behaviors $\left(\epsilon_{x}\right.$ and $\left.v_{x}\right)$ at metal surfaces, are depending on the bulk and surface parameters $\left(k_{F}\right.$ and $W$ ) [27-29], and thus they are created by bulk and surface electrons. When correlation is included, screening effects dump the bulk contribution, such that the asymptotic properties of $\epsilon_{\mathrm{xc}}$ and $v_{\mathrm{xc}}$ are mainly created by a density-independent $\mathrm{xc}$ effect of the surface region, as proved by the SA-TPSS result for the Airy gas. Thus, the Airy-gas model system can be efficiently used in modeling various phenomena outside metal surfaces, even being an alternative to the ad hoc LDA xc potential modifications [18,19].

\section{CONCLUSIONS}

In conclusion, we have derived an exact meta-GGA condition for the correct imagelike surface asymptotics of the xc energy per particle $\varepsilon_{\mathrm{xc}}$ and the $\mathrm{KS}$ xc potential $v_{\mathrm{xc}}$. Our formula [Eq. (5)] depends only on the semilocal ingredient $\alpha$ and takes advantage of the nonlocality of the kinetic energy density beyond the von Weizsäcker term [38]. The existence of this exact condition represents an important contribution in the framework of DFT, as it shows that surface asymptotics can be described by semilocal meta-GGA functionals. On the contrary, no GGA can be constructed that is able to describe correctly the asymptotics of both $\varepsilon_{\mathrm{xc}}$ and $v_{\mathrm{xc}}$. In fact, there is, to our knowledge, no GGA functional that yields a realistic $\mathrm{KS} \mathrm{xc}$ potential at metal surfaces.

We have demonstrated that our exact condition can be easily implemented in any meta-GGA functional, keeping its original accuracy for standard ground-state properties and providing, at the same time, a correct description of the surface asymptotics. We have constructed the SA-TPSS functional, which we have shown to perform as the TPSS for covalent chemistry and to improve over it for noncovalent interactions and surfacerelated problems. This new functional can thus be a promising tool for the investigation of surface-sensitive electronic structure calculations, such as molecule/molecular complexsurface, cluster-surface, and surface-surface interactions.

\section{ACKNOWLEDGMENTS}

We thank TURBOMOLE GmbH for providing the TURBOMOLE program package. We thank Professor J. P. Perdew for useful discussions. L.A.C. thanks the Donostia International Physics Center (DIPC), where this work was started.

\section{APPENDIX A}

In case of semi-infinite jellium surfaces, the one-particle eigenfunctions $\phi_{k_{z}}(z)$ have a continuous energy spectrum $\epsilon_{k_{z}}=V_{\mathrm{KS}}(\infty)+k_{z}^{2} / 2$, and are solutions of the one-dimensional KS equation

$$
\left(-\frac{\partial^{2}}{2 \partial z^{2}}+V_{\mathrm{KS}}(z)-\epsilon_{k_{z}}\right) \phi_{k_{z}}(z)=0 .
$$

Here, $V_{\mathrm{KS}}(z)=V_{H}(z)+V_{\mathrm{xc}}(z)$ is the sum of the total classical electrostatic potential (which incorporates the positive background), and the xc potential.

In case of the Airy gas, the effective potential has the linear form

$$
v_{\mathrm{eff}}(Z)= \begin{cases}-F Z & \text { when }-\infty<\mathrm{Z}<\mathrm{L}, \\ +\infty & \text { when } \mathrm{Z} \geqslant \mathrm{L},\end{cases}
$$

with $F>0$ being the slope of the effective potential [51]. Thus, the one-particle normalized eigenfunctions $\phi_{j}(Z)$ satisfy the 
one-dimensional equation

$$
\begin{array}{r}
\left(-\frac{\partial^{2}}{2 \partial Z^{2}}-F Z-\epsilon_{j}\right) \phi_{j}(Z)=0 \\
\phi_{j}(\infty)=\phi_{j}(L)=0,
\end{array}
$$

being proportional to the Airy function. Here, $Z$ is the distance perpendicular to the surface. It is convenient to consider the scaled distance $z=Z(2 F)^{1 / 3}$ [51], as used in Sec. IV.

\section{APPENDIX B}

The electron density and kinetic energy density of a jellium surface are

$$
\begin{gathered}
\rho(z)=\frac{1}{4 \pi^{2}} \int_{-k_{F}}^{k_{F}} d k_{z}\left(k_{F}^{2}-k_{z}^{2}\right)\left|\phi_{k_{z}}(z)\right|^{2}, \\
\tau(z)=\int_{-k_{F}}^{k_{F}} d k_{z}\left[\frac{1}{8 \pi^{2}}\left|\frac{\partial \phi_{k_{z}}(z)}{\partial z}\right|^{2}\left(k_{F}^{2}-k_{z}^{2}\right)+\frac{1}{16 \pi^{2}}\left|\phi_{k_{z}}(z)\right|^{2}\left(k_{F}^{2}-k_{z}^{2}\right)^{2}\right],
\end{gathered}
$$

with $k_{F}$ being the magnitude of the bulk Fermi wave vector.

Using Eqs. (3) and (4) we obtain, when $z \rightarrow \infty$, the following expressions:

$$
\begin{gathered}
\rho \rightarrow\left(1 / 4 \frac{N^{2} W}{k_{F} \pi^{2} z^{2}}-1 / 8 \frac{N^{2} \sqrt{2} W^{3 / 2}}{k_{F}^{3} z^{3} \pi^{2}}\right) e^{-2 z \sqrt{2 W}} z^{2 \alpha_{\mathrm{KS}}}, \\
\tau \rightarrow 1 / 4 \frac{N^{2} W^{2}}{k_{F} \pi^{2} z^{2} e^{2 z \sqrt{2 W}} z^{-2 \alpha_{\mathrm{KS}}}}+1 / 32 \frac{\left(-8 k_{F}^{4} \sqrt{W} \alpha_{\mathrm{KS}}-4 k_{F}^{2} W^{3 / 2}+12 k_{F}^{4} \sqrt{W}\right) N^{2} W \sqrt{2}}{k_{F}^{5} \pi^{2} z^{3} e^{2 z \sqrt{2 W}} z^{-2 \alpha_{\mathrm{KS}}}} \\
+1 / 32 \frac{\left(-12 k_{F}^{2} W \sqrt{2}+3 \sqrt{2} k_{F}{ }^{4}+4 k_{F}^{2} W \sqrt{2} \alpha_{\mathrm{KS}}-4 \sqrt{2} k_{F}^{4} \alpha_{\mathrm{KS}}+2 \sqrt{2} k_{F}^{4} \alpha_{\mathrm{KS}}{ }^{2}\right) N^{2} W \sqrt{2}}{k_{F}^{5} \pi^{2} z^{4} e^{2 z \sqrt{2 W}} z^{-2 \alpha_{\mathrm{KS}}}} \\
+1 / 32 \frac{\left(6 k_{F}^{2} \sqrt{W} \alpha_{\mathrm{KS}}+6 W^{3 / 2}-2 k_{F}^{2} \sqrt{W} \alpha_{\mathrm{KS}}{ }^{2}-6 k_{F}^{2} \sqrt{W}\right) N^{2} W \sqrt{2}}{k_{F}^{5} \pi^{2} z^{5} e^{2 z \sqrt{2 W}} z^{-2 \alpha_{\mathrm{KS}}}} .
\end{gathered}
$$

Then,

$$
\alpha \rightarrow-\frac{\left(-60 \sqrt[3]{3} \sqrt[6]{2} k_{F}^{2} z \sqrt{W}+40 \sqrt[3]{3} 2^{2 / 3} W-15 \sqrt[3]{3} 2^{2 / 3} k_{F}^{2}\right) e^{4 / 3} z \sqrt{2} \sqrt{W} z^{-4 / 3 \alpha_{\mathrm{KS}}-2 / 3} 2^{2 / 3}}{54 W^{2 / 3} N^{4 / 3} k_{F}^{4 / 3}}
$$

and

$$
F_{x c} \rightarrow \frac{4}{9 \eta} \frac{\left(\pi 2^{2 / 3} 3^{2 / 3} \sqrt[3]{k_{F}}+9 O\left(z^{-4 / 3}\right) N^{2 / 3} \sqrt[3]{W} \sqrt[3]{z}\right) e^{2 / 3 z \sqrt{2} \sqrt{W}} z^{-2 / 3 \alpha_{\mathrm{KS}}-1 / 3}}{N^{2 / 3} \sqrt[3]{W}}
$$

where $N$ is a normalization constant, $W$ is the work function, and $k_{F}$ is the bulk Fermi wave vector. Here, $F_{\mathrm{xc}}=\epsilon_{\mathrm{xc}}^{\mathrm{MGGA}} / \epsilon_{x}^{\mathrm{LDA}}$ is the enhancement factor corresponding to the energy density of Eq. (5).

[1] N. Garcia, B. Reihl, K. H. Frank, and A. R. Williams, Image States: Binding Energies, Effective Masses, and Surface Corrugation, Phys. Rev. Lett. 54, 591 (1985).

[2] S. Datta, Quantum Transport: Atom to Transistor (Cambridge University Press, Cambridge, 2005).

[3] J. Rundgren and G. Malmström, Surface-Resonance Fine Structure in Low-Energy Electron Diffraction, Phys. Rev. Lett. 38, 836 (1977).

[4] G. Binnig, K. H. Frank, H. Fuchs, N. Garcia, B. Reihl, H. Rohrer, F. Salvan, and A. R. Williams, Tunneling Spectroscopy and Inverse Photoemission: Image and Field States, Phys. Rev. Lett. 55, 991 (1985).
[5] J. Pitarke, F. Flores, and P. Echenique, Tunneling spectroscopy: surface geometry and interface potential effects, Surf. Sci. 234, 1 (1990).

[6] C. B. Harris, N.-H. Ge, R. L. L. Jr., J. D. McNeill, and C. M. Wong, Femtosecond dynamics of electrons on surfaces and at interfaces, Annu. Rev. Phys. Chem. 48, 711 (1997).

[7] T. Fauster and W. Steinmann, in Electromagnetic Waves: Recent Development in Research, Vol. 2, edited by P. Halevi (Elsevier, Amsterdam, 1995), p. 350.

[8] N. Lang and W. Kohn, Theory of metal surfaces: Induced surface charge and image potential, Phys. Rev. B 7, 3541 (1973). 
[9] W. Kohn, Nobel lecture: Electronic structure of matter-wave functions and density functionals, Rev. Mod. Phys. 71, 1253 (1999).

[10] W. Kohn and L. J. Sham, Self-consistent equations including exchange and correlation effects, Phys. Rev. 140, A1133 (1965).

[11] O. Gunnarsson, M. Jonson, and B. I. Lundqvist, Descriptions of exchange and correlation effects in inhomogeneous electron systems, Phys. Rev. B 20, 3136 (1979).

[12] A. G. Eguiluz and W. Hanke, Evaluation of the exchangecorrelation potential at a metal surface from many-body perturbation theory, Phys. Rev. B 39, 10433 (1989).

[13] A. G. Eguiluz, M. Heinrichsmeier, A. Fleszar, and W. Hanke, First-Principles Evaluation of the Surface Barrier for a Kohn-Sham Electron at a Metal Surface, Phys. Rev. Lett. 68, 1359 (1992).

[14] I. D. White, R. W. Godby, M. M. Rieger, and R. J. Needs, Dynamic Image Potential at an al(111) Surface, Phys. Rev. Lett. 80, 4265 (1998).

[15] Z. Qian and V. Sahni, Exact electronic properties in the classically forbidden region of a metal surface, Int. J. Quantum Chem. 104, 929 (2005).

[16] J.-T. Hoeft, M. Kittel, M. Polcik, S. Bao, R. L. Toomes, J.-H. Kang, D. P. Woodruff, M. Pascal, and C. L. A. Lamont, Molecular Adsorption Bond Lengths at Metal Oxide Surfaces: Failure of Current Theoretical Methods, Phys. Rev. Lett. 87, 086101 (2001).

[17] A. D. Becke, Density-functional exchange-energy approximation with correct asymptotic behavior, Phys. Rev. A 38, 3098 (1988).

[18] P. A. Serena, J. M. Soler, and N. García, Self-consistent image potential in a metal surface, Phys. Rev. B 34, 6767 (1986).

[19] E. Chulkov, V. Silkin, and P. Echenique, Image potential states on metal surfaces: Binding energies and wave functions, Surf. Sci. 437, 330 (1999).

[20] O. Gunnarsson and R. O. Jones, Density functional calculations for atoms, molecules and clusters, Phys. Scr. 21, 394 (1980).

[21] S. Ossicini, C. M. Bertoni, and P. Gies, Image plane for surface potential, Europhys. Lett. 1, 661 (1986).

[22] P. García-González, J. E. Alvarellos, E. Chacón, and P. Tarazona, Image potential and the exchange-correlation weighted density approximation functional, Phys. Rev. B 62, 16063 (2000).

[23] J. Harris and A. Griffin, Correlation energy and van der waals interaction of coupled metal films, Phys. Rev. B 11, 3669 (1975).

[24] D. C. Langreth and J. P. Perdew, Exchange-correlation energy of a metallic surface: Wave-vector analysis, Phys. Rev. B 15, 2884 (1977).

[25] O. Gunnarsson and B. I. Lundqvist, Exchange and correlation in atoms, molecules, and solids by the spin-density-functional formalism, Phys. Rev. B 13, 4274 (1976).

[26] L. A. Constantin and J. M. Pitarke, Adiabatic-connectionfluctuation-dissipation approach to long-range behavior of exchange-correlation energy at metal surfaces: A numerical study for jellium slabs, Phys. Rev. B 83, 075116 (2011).

[27] C. M. Horowitz, L. A. Constantin, C. R. Proetto, and J. M. Pitarke, Position-dependent exact-exchange energy for slabs and semi-infinite jellium, Phys. Rev. B 80, 235101 (2009).

[28] Z. Qian, Asymptotic behavior of the kohn-sham exchange potential at a metal surface, Phys. Rev. B 85, 115124 (2012).
[29] C. M. Horowitz, C. R. Proetto, and J. M. Pitarke, Localized versus extended systems in density functional theory: Some lessons from the kohn-sham exact exchange potential, Phys. Rev. B 81, 121106 (2010).

[30] C. M. Horowitz, C. R. Proetto, and S. Rigamonti, Kohn-Sham Exchange Potential for a Metallic Surface, Phys. Rev. Lett. 97, 026802 (2006).

[31] C. M. Horowitz, C. R. Proetto, and J. M. Pitarke, Exact-exchange kohn-sham potential, surface energy, and work function of jellium slabs, Phys. Rev. B 78, 085126 (2008).

[32] L.-H. Ye, Surface calculations with asymptotically long-ranged potentials in the full-potential linearized augmented plane-wave method, Phys. Rev. B 92, 115132 (2015).

[33] E. Engel, Exact exchange plane-wave-pseudopotential calculations for slabs, J. Chem. Phys. 140, 18A505 (2014).

[34] E. Engel, Asymptotic behavior of exact exchange potential of slabs, Phys. Rev. B 89, 245105 (2014).

[35] E. Engel, J. Chevary, L. Macdonald, and S. Vosko, Asymptotic properties of the exchange energy density and the exchange potential of finite systems: Relevance for generalized gradient approximations, Z. Phys. D 23, 7 (1992).

[36] R. van Leeuwen and E. J. Baerends, Exchange-correlation potential with correct asymptotic behavior, Phys. Rev. A 49, 2421 (1994).

[37] R. Armiento and S. Kümmel, Orbital Localization, Charge Transfer, and Band Gaps in Semilocal Density-Functional Theory, Phys. Rev. Lett. 111, 036402 (2013).

[38] F. Della Sala, E. Fabiano, and L. A. Constantin, Kohn-sham kinetic energy density in the nuclear and asymptotic regions: Deviations from the von weizsäcker behavior and applications to density functionals, Phys. Rev. B 91, 035126 (2015).

[39] N. D. Lang and W. Kohn, Theory of metal surfaces: Charge density and surface energy, Phys. Rev. B 1, 4555 (1970).

[40] N. Lang and W. Kohn, Theory of metal surfaces: work function, Phys. Rev. B 3, 1215 (1971).

[41] B. Silvi and A. Savin, Classification of chemical bonds based on topological analysis of electron localization functions, Nature (London) 371, 683 (1994).

[42] A. V. Arbuznikov, M. Kaupp, V. G. Malkin, R. Reviakine, and O. L. Malkina, Validation study of meta-gga functionals and of a model exchange-correlation potential in density functional calculations of epr parameters, Phys. Chem. Chem. Phys. 4, 5467 (2002).

[43] J. Tao, J. P. Perdew, V. N. Staroverov, and G. E. Scuseria, Climbing the Density Functional Ladder: Nonempirical Meta generalized Gradient Approximation Designed for Molecules and Solids, Phys. Rev. Lett. 91, 146401 (2003).

[44] L. A. Constantin, A. Terentjevs, F. Della Sala, and E. Fabiano, Gradient-dependent upper bound for the exchange-correlation energy and application to density functional theory, Phys. Rev. B 91, 041120 (2015).

[45] D. V. Feinblum, J. Kenison, and K. Burke, Communication: Testing and using the lewin-lieb bounds in density functional theory, J. Chem. Phys. 141, 241105 (2014).

[46] B. Wood, N. D. M. Hine, W. M. C. Foulkes, and P. GarcíaGonzález, Quantum monte carlo calculations of the surface energy of an electron gas, Phys. Rev. B 76, 035403 (2007).

[47] J. P. Perdew and Y. Wang, Accurate and simple analytic representation of the electron-gas correlation energy, Phys. Rev. B 45, 13244 (1992). 
[48] J. P. Perdew, K. Burke, and M. Ernzerhof, Generalized Gradient Approximation Made Simple, Phys. Rev. Lett. 77, 3865 (1996).

[49] L. A. Constantin, E. Fabiano, and F. D. Sala, Metagga exchange-correlation functional with a balanced treatment of nonlocality, J. Chem. Theory Comput. 9, 2256 (2013).

[50] E. Fabiano, L. A. Constantin, and F. Della Sala, Testing the broad applicability of the pbeint gga functional and its one-parameter hybrid form, Int. J. Quantum Chem. 113, 673 (2013).

[51] W. Kohn and A. E. Mattsson, Edge Electron Gas, Phys. Rev. Lett. 81, 3487 (1998).
[52] R. Armiento and A. E. Mattsson, Functional designed to include surface effects in self-consistent density functional theory, Phys. Rev. B 72, 085108 (2005)

[53] L. Vitos, B. Johansson, J. Kollár, and H. L. Skriver, Local kinetic-energy density of the airy gas, Phys. Rev. A 61, 052511 (2000).

[54] L. A. Constantin and A. Ruzsinszky, Kinetic energy density functionals from the airy gas with an application to the atomization kinetic energies of molecules, Phys. Rev. B 79, 115117 (2009).

[55] L. Vitos, B. Johansson, J. Kollár, and H. L. Skriver, Exchange energy in the local airy gas approximation, Phys. Rev. B 62, 10046 (2000). 